\title{
On Schrödinger equations with modified dispersion
}

\author{
Rémi Carles
}

Communicated by Yvan Martel, received May 31, 2011.

\begin{abstract}
We consider the nonlinear Schrödinger equation with a modified spatial dispersion, given either by an homogeneous Fourier multiplier, or by a bounded Fourier multiplier. Arguments based on ordinary differential equations yield ill-posedness results which are sometimes sharp. When the Fourier multiplier is bounded, we infer that no Strichartztype estimate improving on Sobolev embedding is available. Finally, we show that when the symbol is bounded, the Cauchy problem may be ill-posed in the case of critical regularity, with arbitrarily small initial data. The same is true when the symbol is homogeneous of degree one, where scaling arguments may not even give the right critical value.
\end{abstract}

\section{CONTENTS}

1. Introduction 173

2. Proof of Theorem $\overline{1.2} \quad 177$

3. Proof of Corollary $\overline{1.4} \quad 179$

4. Ill-posedness 181

References 182

\section{Introduction}

For $(t, x) \in \mathbf{R}_{+} \times \mathbf{R}^{d}$, we consider

$$
i \partial_{t} u+P(D) u=\lambda|u|^{2 \sigma} u \quad ; \quad u_{\mid t=0}=u_{0},
$$

where $D=-i \nabla_{x}, P: \mathbf{R}^{d} \rightarrow \mathbf{R}, \lambda \in \mathbf{R}$ and $\sigma>0$. Since the Fourier multiplier $P$ is real-valued, the free flow $(\lambda=0)$ generates a unitary group on $\dot{H}^{s}\left(\mathbf{R}^{d}\right), s \in \mathbf{R}$ :

$$
S(t)=e^{-i t P(D)} .
$$

We study the local well-posedness for the Cauchy problem (1.1), defined as follows:

1991 Mathematics Subject Classification. Primary 35Q55; Secondary 35A01, 35B33, 35B45.

Key words and phrases. Nonlinear Schrödinger equation, Fourier multiplier, ill-posed.

This work was supported by the French ANR project R.A.S. (ANR-08-JCJC-0124-01). 
DEFINITION 1.1 (From [22]). Let $s \in \mathbf{R}$. The Cauchy problem (1.1) is well posed in $H^{s}\left(\mathbf{R}^{d}\right)$ if, for all bounded subset $B \subset H^{s}\left(\mathbf{R}^{d}\right)$, there exist $T>0$ and a Banach space $X_{T}$ continuously embedded into $C\left([0, T] ; H^{s}\left(\mathbf{R}^{d}\right)\right)$ such that for all $u_{0} \in H^{s}\left(\mathbf{R}^{d}\right)$, 1.1. has a unique solution $u \in X_{T}$, and the mapping $u_{0} \mapsto u$ is uniformly continuous from $\left(B,\|\cdot\|_{H^{s}}\right)$ to $C\left([0, T] ; H^{s}\left(\mathbf{R}^{d}\right)\right)$.

Note that this definition is not quite universal, since for instance the uniformity on balls rules out the critical cases. This definition is well suited for cases where the solution is constructed by a fixed point argument, in a ball of a Banach space, whose radius is related to the size of the initial data; in critical cases, not only the size of the initial data comes into play, but also the profile of the initial data. We also point out that even in the case of Schrödinger equations $\left(P(\xi)=-|\xi|^{2}\right)$, continuity is known only in a limited number of cases (and implies uniform continuity in the above sense): see [28] for $s=0$, [19] for $s=1$ and $s=2$, and [9] for $0<s<1$. However, when we deny the above notion of local well-posedness, we will do so in a rather explicit way, in the same fashion as in [8, 11].

We consider two cases in 1.1.

- $P$ is homogeneous, $P(\mu \xi)=\mu^{m} P(\xi)$, for all $\xi \in \mathbf{R}^{d}, \mu>0$, with $m \geqslant 1$.

- $P$ is bounded, $P \in L^{\infty}\left(\mathbf{R}^{d}\right)$.

The first case includes the standard Schrödinger operator $\left(P(\xi)=-|\xi|^{2}\right)$, and the fourthorder Schrödinger operator $\left(P(\xi)=|\xi|^{4}\right)$, studied for instance in [2, 25, 26]. Smoothing effects and dispersive estimates have been established for rather general Fourier multipliers $P$ in [4, 20]. The case $d=1, P(\xi)=\xi^{2 j+1}, j \in \mathbf{N}$, has been studied initially in [21]; the case $d=2$, with $P$ a polynomial of degree $m=3$ has been studied in [3], revealing different dispersive phenomena according to the precise structure of $P$. The case $m \in 2 \mathbf{N}$, with $P$ elliptic and $\nabla^{2} P$ non-degenerate outside $\{\xi=0\}$, appears as a particular case of the framework in [15]. It is shown there that if $s>\max \left(0, s_{0}\right)$, then the Cauchy problem 1.1. is locally well-posed in $H^{s}\left(\mathbf{R}^{d}\right)$, where

$$
s_{0}=\frac{d}{2}-\frac{m}{2 \sigma} .
$$

This index corresponds to the one given by scaling arguments: if $u$ solves 1.1, then for any $\Lambda>0, u_{\Lambda}:(t, x) \mapsto \Lambda^{m /(2 \sigma)} u\left(\Lambda^{m} t, \Lambda x\right)$ solves the same equation. The value of $s$ for which the $\dot{H}^{s}\left(\mathbf{R}^{d}\right)$-norm is invariant under $u \mapsto u_{\Lambda}$ is $s=s_{0}$. We will see in 1.3 that this scaling argument may not yield the sharp Sobolev regularity: if $m=1$, the Cauchy problem (1.1) may be strongly ill-posed in $H^{s}\left(\mathbf{R}^{d}\right)$ for all $s \leqslant d / 2$.

In [15], the proof of local well-posedness uses dispersive and Strichartz estimates for $S$, established in [13] for $d=1$, and in [14] for $d \geqslant 2$. Note that in the case $d=1$, dispersive and Strichartz estimates for $S$ are proved in $\left[\mathbf{1 8} \mid\right.$ for $P(\xi)=|\xi|^{m}$ and $m \geqslant 2$ (not necessarily an integer). By resuming arguments similar to those presented in [8, 11], we prove that in this framework, the index $s_{0}$ is sharp, in the sense that the nonlinear flow map fails to be uniformly continuous at the origin in $H^{s}\left(\mathbf{R}^{d}\right)$, if $s<s_{0}$. This property has been established in [26] for the case $P(\xi)=|\xi|^{4}$ with $(d, \sigma)=(3,1)$. However, the index $s_{0}$ may not correspond to the critical Sobolev regularity (see 1.3.

The second case, where $P$ is bounded, is motivated by the results presented in [16], where $P(\xi)=-\frac{1}{h} \arctan \left(h|\xi|^{2}\right)$ is considered to construct numerical approximations of the solution to the linear Schrödinger equation

$$
i \partial_{t} u+\Delta u=V(x) u
$$


and $0<h \ll 1$ denotes the time step. We will see below that in such a framework, no Strichartz estimate is available, even if one is ready to pay some loss of derivative. Another example of bounded symbol one may think of is

$$
i \partial_{t} u+\Delta(1-\Delta)^{-1} u=\lambda|u|^{2 \sigma} u,
$$

which is a possible envelope equation in geometric optics (see e.g. [12, 23]). In these two examples, $P$ is elliptic. We will see however that no Strichartz estimate (better than Sobolev embedding) is available there, and that the critical regularity is $s_{c}=d / 2$.

1.1. Norm inflation. Our result in this direction is:

THEOREM 1.2. Let $d \geqslant 1, \lambda \in \mathbf{R}, \sigma>0$. Assume that either $\sigma$ is an integer, or that there exists an integer $r$ such that $2 \sigma \geqslant r>d / 2$. If $\sigma \in \mathbf{N}$, fix an integer $r>d / 2$.

1. Suppose that $P$ is $m$-homogeneous, with $m \geqslant 1$, and denote $s_{0}=d / 2-m /(2 \sigma)$. Suppose that $s_{0}>0$ and let $0<s<s_{0}$. There exists a family $\left(u_{0}^{h}\right)_{0<h \leqslant 1}$ in $\mathcal{S}\left(\mathbf{R}^{d}\right)$ with

$$
\left\|u_{0}^{h}\right\|_{H^{s}\left(\mathbf{R}^{d}\right)} \rightarrow 0 \text { as } h \rightarrow 0,
$$

$0<t^{h} \rightarrow 0$, and a strong solution $u^{h} \in C\left(\left[0, t^{h}\right] ; H^{r}\left(\mathbf{R}^{d}\right)\right)$ to [1.1, such that:

$$
\left\|u^{h}\left(t^{h}\right)\right\|_{H^{s}\left(\mathbf{R}^{d}\right)} \rightarrow+\infty \text { as } h \rightarrow 0 .
$$

2. If $P$ is bounded, then the above conclusion remains true for any $0<s<d / 2$.

Theorem 1.2 is proved in 4 by adapting the ordinary differential equation mechanism used in, e.g., [8] 11. However, the critical Sobolev regularity $s_{c}$ may satisfy $s_{c}>s_{0}$ (see 1.3): in view of [15] and Theorem 1.2] we have $s_{c}=s_{0}$ at least when $P(\xi)=\mu|\xi|^{m}$, $\mu \in \mathbf{R} \backslash\{0\}, m \in 2 \mathbf{N} \backslash\{0\}$. We also refer to [6] where a different result concerning the lack of well-posedness is established for a broad variety of dispersive equations, even in the linear case.

1.2. Absence of Strichartz estimates. In this paragraph, we focus our discussion on the case where $P$ is bounded. For $s>d / 2, H^{s}\left(\mathbf{R}^{d}\right)$ being an algebra, local well-posedness in $H^{s}\left(\mathbf{R}^{d}\right)$ is straightforward, provided that the nonlinearity is sufficiently smooth (see e.g. [27]). Therefore, the critical threshold is $s_{c}=d / 2$, and from Theorem 1.2 no dispersive property is present to decrease this number. More precisely, no Strichartz estimate is available for $S(\cdot)$, even if one is ready to pay some loss of regularity which is not worse than the result provided by Sobolev embedding. To state this property precisely, we recall the standard definition.

Definition 1.3. A pair $(p, q) \neq(2, \infty)$ is admissible if $p \geqslant 2, q \geqslant 2$, and

$$
\frac{2}{p}=d\left(\frac{1}{2}-\frac{1}{q}\right) \text {. }
$$

By Sobolev embedding, for all $(p, q)$ (not necessarily admissible) with $2 \leqslant q<\infty$, there exists $C>0$ such that for all $u_{0} \in H^{d / 2-d / q}\left(\mathbf{R}^{d}\right)$, and all finite time interval $I$,

$$
\begin{aligned}
\left\|S(\cdot) u_{0}\right\|_{L^{p}\left(I ; L^{q}\left(\mathbf{R}^{d}\right)\right)} & \leqslant C\left\|S(\cdot) u_{0}\right\|_{L^{p}\left(I ; H^{d / 2-d / q}\left(\mathbf{R}^{d}\right)\right)} \\
& \leqslant C\left\|u_{0}\right\|_{L^{p}\left(I ; H^{d / 2-d / q}\left(\mathbf{R}^{d}\right)\right)}=C|I|^{1 / p}\left\|u_{0}\right\|_{H^{d / 2-d / q}\left(\mathbf{R}^{d}\right)} .
\end{aligned}
$$

When $P$ is bounded, this estimate cannot be improved: 
COROLlary 1.4. Let $d \geqslant 1$, and $P \in L^{\infty}\left(\mathbf{R}^{d} ; \mathbf{R}\right)$. Suppose that there exist an admissible pair $(p, q)$, an index $k \in \mathbf{R}$, a time interval $I \ni 0,|I|>0$, and a constant $C>0$ such that

$$
\left\|S(\cdot) u_{0}\right\|_{L^{p}\left(I ; L^{q}\left(\mathbf{R}^{d}\right)\right)} \leqslant C\left\|u_{0}\right\|_{H^{k}\left(\mathbf{R}^{d}\right)}, \quad \forall u_{0} \in H^{k}\left(\mathbf{R}^{d}\right) .
$$

Then necessarily, $k \geqslant 2 / p=d / 2-d / q$.

The fact that no standard Strichartz estimate (with no loss) is available for $S(\cdot)$ is rather clear, since the dispersion relation is given by $\tau=P(\xi)$, and defines a characteristic variety which is bounded in $\tau$. However, one could expect the existence of Strichartz estimates with loss of regularity, in the same fashion as in [1, 5, 7] (where the geometric framework - the space variable belongs to a compact manifold - rules out the existence of the standard dispersive properties). The fact that this is not so is a rather direct consequence of Theorem 1.2 (where $\sigma>0$ is arbitrary), and of the argument given in [7] to prove Proposition 3.1. It may seem surprising to prove Corollary 1.4 as a consequence of a nonlinear analysis; we insist on the fact that the proof of Theorem 1.2 is rather simple, and the deduction of Corollary 1.4 involves another nonlinear result, whose proof is also quite short (see Proposition 3.1 below).

1.3. Critical cases. In $|\mathbf{1 0}|$, local well-posedness in $H^{s_{c}}\left(\mathbf{R}^{d}\right)$ for small data is established for Equation (1.1) in the case $P(\xi)=-|\xi|^{2}$, where

$$
s_{c}=\frac{d}{2}-\frac{1}{\sigma}
$$

coincides with $s_{0}$ in that case, since $m=2$. In [24], local well-posedness in $H^{d / 2}\left(\mathbf{R}^{d}\right)$ for small data is established for the same operator, with nonlinearities which are allowed to grow exponentially. In these two papers, the proof uses Strichartz estimates (in Besov spaces). On the other hand, when $P$ is bounded, the Cauchy problem may be ill-posed in $H^{d / 2}\left(\mathbf{R}^{d}\right)$, even for nonlinearities growing algebraically. Moreover, when $P$ is $m$ homogeneous with $m=1$, the critical Sobolev regularity may not be $s_{0}$, but $s_{c}=d / 2>$ $s_{0}$, with ill-posedness for $s=s_{c}$.

Proposition 1.5. Let $\lambda \in \mathbf{R} \backslash\{0\}, \sigma>0$. Assume that either $\sigma$ is an integer, or that there exists an integer $r$ such that $2 \sigma \geqslant r>d / 2$. In either of the two cases:

- $P(\xi)=c \cdot \xi, c \in \mathbf{R}^{d}$, or

- $P$ is constant,

for all $\delta>0$, there exists $u_{0} \in H^{d / 2}\left(\mathbf{R}^{d}\right)$ with $\left\|u_{0}\right\|_{H^{d / 2}\left(\mathbf{R}^{d}\right)} \leqslant \delta$ such that (1.1) has a solution $u \in C\left(\mathbf{R}_{+} ; \mathcal{D}^{\prime}\left(\mathbf{R}^{d}\right)\right)$, and for any $t>0, u(t, \cdot) \notin H^{d / 2}\left(\mathbf{R}^{d}\right)$.

For comparison with other results, note that in the first case, $P$ is not elliptic if $d \geqslant 2$. In the second case, $P \neq 0$ is elliptic, but $\nabla^{2} P=0$ is obviously degenerate.

Unlike what happens in the presence of Strichartz estimates ([10, 24]), this result yields examples where local well-posedness fails in the critical case $s=s_{c}$, even for small data. We prove Proposition 1.5 in 4 we present the cases $d=2, \sigma>0$, and $d \geqslant 1$, $\sigma=1 / 2$, only, for the convenience of the exposition, but the argument can be extended to any space dimension, up to more intricate computations.

In the case $m=1$ (at least), the mere assumption that $P$ is $m$-homogeneous is not enough to characterize the critical Sobolev regularity in 1.1., or the existence of Strichartz estimates. Indeed, the symbol $P(\xi)=|\xi|$ corresponds to the wave equation, for which Strichartz estimates are available when $d \geqslant 2$, and so $s_{c}<d / 2$. See e.g. [17]. This 
suggests that also when $m>1$, the value $s_{0}$ may not be sharp when $P$ is not proportional to $|\xi|^{m}$, but for instance of the form $|\xi|^{m-1} c \cdot \xi, c \in \mathbf{R}^{d}$, or more generally when $P$ is not elliptic; see also [3, 4, 20] for remarks in this direction.

\section{Proof of Theorem 1.2}

The proof of Theorem 1.2 proceeds along the same lines as in [8 11] (see also [27]). Fix $s$ as in Theorem 1.2 Consider initial data of the form

$$
u_{0}^{h}(x)=h^{s-d / 2} \kappa^{h} a_{0}\left(\frac{x}{h}\right),
$$

with $0<h \ll 1, a_{0}(x)=e^{-|x|^{2}}$, and

$$
\kappa^{h}=\left(\log \frac{1}{h}\right)^{-\theta}
$$

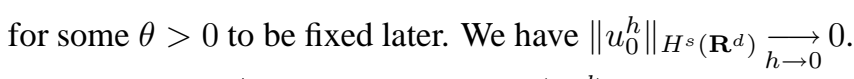

Fix $r>d / 2$ an integer: in $H^{r}\left(\mathbf{R}^{d}\right)$, the nonlinearity can be viewed as a perturbation, and since $a_{0} \in \mathcal{S}\left(\mathbf{R}^{d}\right)$, there exists $T^{h}>0$ and a unique, strong, solution $u^{h} \in C\left(\left[0, T^{h}\right] ; H^{r}\left(\mathbf{R}^{d}\right)\right)$ to (1.1] (see e.g. [27]). At this stage, we have no estimate on the dependence of $T^{h}$ upon $h$ as $h \rightarrow 0$. Introduce the scaling

$$
\psi(\tau, y)=h^{d / 2-s} u^{h}\left(h^{2+\alpha} \tau, h y\right),
$$

for some $\alpha$ to be precised later:

$$
\|\psi(\tau)\|_{\dot{H}^{s}\left(\mathbf{R}^{d}\right)}=\left\|u^{h}\left(h^{2+\alpha} \tau\right)\right\|_{\dot{H}^{s}\left(\mathbf{R}^{d}\right)} .
$$

Denote $\varepsilon=h^{2 \sigma(d / 2-s)-2-\alpha}$. The function $\psi$ solves the Cauchy problem

$$
i \varepsilon \partial_{\tau} \psi+h^{2 \sigma(d / 2-s)} P\left(h^{-1} D_{y}\right) \psi=\lambda|\psi|^{2 \sigma} \psi ; \quad \psi_{\mid \tau=0}=\kappa^{h} a_{0} .
$$

2.1. Choice of $\alpha$. When $P$ is $m$-homogeneous, Equation (2.1) simplifies to

$$
i \varepsilon \partial_{\tau} \psi+h^{2 \sigma(d / 2-s)-m} P\left(D_{y}\right) \psi=\lambda|\psi|^{2 \sigma} \psi ; \quad \psi_{\mid \tau=0}=\kappa^{h} a_{0} .
$$

For $\omega>0$, we set

$$
2+\alpha=\frac{1}{m+\omega}\left((m-1+\omega) 2 \sigma\left(\frac{d}{2}-s\right)+m\right),
$$

in which case we have:

$$
\varepsilon=h^{2 \sigma\left(s_{0}-s\right) /(m+\omega)} .
$$

Therefore, $\varepsilon \rightarrow 0$ as $h \rightarrow 0$ since $s<s_{0}$. We also compute

$$
h^{2 \sigma(d / 2-s)-m}=\varepsilon^{m+\omega} .
$$

When $P$ is bounded, we consider $2+\alpha=\sigma(d / 2-s)$. Therefore,

$$
2 \sigma\left(\frac{d}{2}-s\right)-2-\alpha>0 \quad \text { (hence } \varepsilon \rightarrow 0 \text { as } h \rightarrow 0 \text { ), } \quad \text { and } 2+\alpha>0 .
$$


2.2. The ODE approximation. Introduce the solution to

$$
i \varepsilon \partial_{\tau} \varphi=\lambda|\varphi|^{2 \sigma} \varphi ; \quad \varphi_{\mid \tau=0}=\kappa^{h} a_{0} .
$$

It is given by

$$
\varphi(\tau, y)=\kappa^{h} a_{0}(y) \exp \left(-i \lambda \frac{\tau}{\varepsilon}\left(\kappa^{h}\right)^{2 \sigma}\left|a_{0}(y)\right|^{2 \sigma}\right) .
$$

Since $a_{0}$ is a Gaussian, $\varphi \in C^{\infty}\left(\mathbf{R} \times \mathbf{R}^{d}\right)$ regardless of $\sigma>0$, and for any $r \geqslant 0$,

$$
\|\varphi(\tau)\|_{H^{r}\left(\mathbf{R}^{d}\right)} \lesssim\left(\kappa^{h}\right)^{1+2 \sigma r}\left(\frac{\tau}{\varepsilon}\right)^{r}+\kappa^{h} .
$$

LEMMA 2.1. Let $r>d / 2$ be an integer, with in addition $r \leqslant 2 \sigma$ if $\sigma \notin \mathbf{N}$. In either of the cases of Theorem 1.2 we can find $\delta>0$ independent of $\theta>0$ such that for $\varepsilon>0$ sufficiently small, $\psi \in C\left(\left[0, \varepsilon(\log 1 / \varepsilon)^{\delta}\right] ; H^{r}\left(\mathbf{R}^{d}\right)\right)$, and

$$
\sup _{0 \leqslant \tau \leqslant \varepsilon\left(\log \frac{1}{\varepsilon}\right)^{\delta}}\|\psi(\tau)-\varphi(\tau)\|_{H^{r}\left(\mathbf{R}^{d}\right) \underset{\varepsilon \rightarrow 0}{\longrightarrow} 0 .}^{\longrightarrow}
$$

Proof. Denote by $w=\psi-\varphi$ the error. It solves

$$
\begin{aligned}
i \varepsilon \partial_{\tau} w+h^{2 \sigma(d / 2-s)} P\left(h^{-1} D_{y}\right) w= & h^{2 \sigma(d / 2-s)} P\left(h^{-1} D_{y}\right) \varphi \\
& +\lambda\left(|w+\varphi|^{2 \sigma}(w+\varphi)-|\varphi|^{2 \sigma} \varphi\right),
\end{aligned}
$$

with $w_{\mid \tau=0}=0$. Using the facts that $P$ is real-valued, $z \mapsto|z|^{2 \sigma} z$ is sufficiently smooth, and $H^{r}\left(\mathbf{R}^{d}\right)$ is an algebra, we find

$$
\begin{aligned}
\|w(\tau)\|_{H^{r}\left(\mathbf{R}^{d}\right)} \lesssim & \frac{1}{\varepsilon} \int_{0}^{\tau}\left\|h^{2 \sigma(d / 2-s)} P\left(h^{-1} D_{y}\right) \varphi\left(\tau^{\prime}\right)\right\|_{H^{r}\left(\mathbf{R}^{d}\right)} d \tau^{\prime} \\
& +\frac{1}{\varepsilon} \int_{0}^{\tau}\left(\left\|w\left(\tau^{\prime}\right)\right\|_{H^{r}\left(\mathbf{R}^{d}\right)}^{2 \sigma}+\left\|\varphi\left(\tau^{\prime}\right)\right\|_{H^{r}\left(\mathbf{R}^{d}\right)}^{2 \sigma}\right)\left\|w\left(\tau^{\prime}\right)\right\|_{H^{r}\left(\mathbf{R}^{d}\right)} d \tau^{\prime} .
\end{aligned}
$$

In the case where $P$ is $m$-homogeneous, we have

$$
\begin{aligned}
\left\|h^{2 \sigma(d / 2-s)} P\left(h^{-1} D_{y}\right) \varphi\left(\tau^{\prime}\right)\right\|_{H^{r}\left(\mathbf{R}^{d}\right)} & \lesssim h^{2 \sigma(d / 2-s)-m}\left\|\varphi\left(\tau^{\prime}\right)\right\|_{H^{r+m}\left(\mathbf{R}^{d}\right)} \\
& \lesssim h^{2 \sigma\left(s_{0}-s\right)}\left\|\varphi\left(\tau^{\prime}\right)\right\|_{H^{r+m}\left(\mathbf{R}^{d}\right)} .
\end{aligned}
$$

In the case where $P$ is bounded, we have

$$
\begin{aligned}
\left\|h^{2 \sigma(d / 2-s)} P\left(h^{-1} D_{y}\right) \varphi\left(\tau^{\prime}\right)\right\|_{H^{r}\left(\mathbf{R}^{d}\right)} & \lesssim h^{2 \sigma(d / 2-s)}\left\|\varphi\left(\tau^{\prime}\right)\right\|_{H^{r}\left(\mathbf{R}^{d}\right)} \\
& \lesssim h^{2 \sigma\left(s_{0}-s\right)}\left\|\varphi\left(\tau^{\prime}\right)\right\|_{H^{r}\left(\mathbf{R}^{d}\right)},
\end{aligned}
$$

where we set $s_{0}=d / 2$ in this case.

In both cases, we check that there exists $\beta>0$ (independent of $\theta$ ) such that

$$
h^{2 \sigma\left(s_{0}-s\right)}=\varepsilon^{1+\beta} .
$$

It is given by the formula

$$
\beta=\frac{2 \sigma\left(s_{0}-d / 2\right)+2+\alpha}{2 \sigma(d / 2-s)-2-\alpha} .
$$

In the homogeneous case, this formula becomes $\beta=m-1+\omega$, and when $P$ is bounded, $\beta=1$. Therefore, in view of (2.2) and since $\kappa^{h} \leqslant 1$, there exist $\beta, \gamma>0$ such that

$$
\left\|h^{2 \sigma(d / 2-s)} P\left(h^{-1} D_{y}\right) \varphi\left(\tau^{\prime}\right)\right\|_{H^{r}\left(\mathbf{R}^{d}\right)} \leqslant \varepsilon^{1+\beta}\left(\left(\frac{\tau}{\varepsilon}\right)^{\gamma}+1\right) .
$$


So long as $\|w(\tau)\|_{H^{r}\left(\mathbf{R}^{d}\right)} \leqslant 1$, with $\tau$ as above, we infer from (2.2):

$$
\|w(\tau)\|_{H^{r}\left(\mathbf{R}^{d}\right)} \lesssim \int_{0}^{\tau} \varepsilon^{\beta}\left(\left(\frac{\tau^{\prime}}{\varepsilon}\right)^{\gamma}+1\right) d \tau^{\prime}+\frac{1}{\varepsilon} \int_{0}^{\tau}\left(1+\left(\frac{\tau^{\prime}}{\varepsilon}\right)^{r}\right)\left\|w\left(\tau^{\prime}\right)\right\|_{H^{r}\left(\mathbf{R}^{d}\right)} d \tau^{\prime} .
$$

Gronwall's Lemma yields

$$
\|w(\tau)\|_{H^{r}\left(\mathbf{R}^{d}\right)} \lesssim \varepsilon^{\beta}\left(\left(\frac{\tau}{\varepsilon}\right)^{\gamma}+1\right) e^{C \tau / \varepsilon+C(\tau / \varepsilon)^{r+1}} \lesssim \varepsilon^{\beta} e^{2 C \tau / \varepsilon+C(\tau / \varepsilon)^{r+1}} .
$$

By choosing $\delta>0$ sufficiently small, the right hand side is controlled by, say, $\varepsilon^{\beta / 2}$, for all $0 \leqslant \tau \leqslant \varepsilon\left(\log \frac{1}{\varepsilon}\right)^{\delta}$. The condition $\|w(\tau)\|_{H^{r}\left(\mathbf{R}^{d}\right)} \leqslant 1$ is verified for such times $\tau$, provided that $\varepsilon$ is sufficiently small. This bootstrap argument implies in particular that for $\varepsilon$ sufficiently small, $\psi \in C\left(\left[0, \varepsilon(\log 1 / \varepsilon)^{\delta}\right] ; H^{r}\left(\mathbf{R}^{d}\right)\right)$, which is not obvious from the very start. The lemma follows.

2.3. Conclusion. Let $r>d / 2$ as in Lemma 2.1 With $\delta>0$ given by Lemma 2.1 we have, since $s<d / 2$ :

$$
\begin{aligned}
\left\|u^{h}\left(h^{2+\alpha} \varepsilon\left(\log \frac{1}{\varepsilon}\right)^{\delta}\right)\right\|_{H^{s}\left(\mathbf{R}^{d}\right)} & \geqslant \varphi\left(\varepsilon\left(\log \frac{1}{\varepsilon}\right)^{\delta}\right) \|_{H^{s}\left(\mathbf{R}^{d}\right)} \\
& -C\left\|\varphi\left(\varepsilon\left(\log \frac{1}{\varepsilon}\right)^{\delta}\right)-\psi\left(\varepsilon\left(\log \frac{1}{\varepsilon}\right)^{\delta}\right)\right\|_{H^{r}\left(\mathbf{R}^{d}\right)} .
\end{aligned}
$$

On the other hand, similar to 2.2, we have:

$$
\|\varphi(\tau)\|_{H^{s}\left(\mathbf{R}^{d}\right)} \gtrsim\left(\kappa^{h}\right)^{1+2 \sigma s}\left(\frac{\tau}{\varepsilon}\right)^{s}-C \kappa^{h},
$$

and so,

$$
\left\|\varphi\left(\varepsilon\left(\log \frac{1}{\varepsilon}\right)^{\delta}\right)\right\|_{H^{s}\left(\mathbf{R}^{d}\right)} \geqslant C\left(\log \frac{1}{\varepsilon}\right)^{s \delta-\theta-2 \sigma \theta s}-o(1) .
$$

For $\theta>0$ sufficiently small, $s \delta-\theta-2 \sigma \theta s>0$, and Lemma2.1 yields

$$
\left\|u^{h}\left(h^{2+\alpha} \varepsilon\left(\log \frac{1}{\varepsilon}\right)^{\delta}\right)\right\|_{H^{s}\left(\mathbf{R}^{d}\right)} \underset{h \rightarrow 0}{\longrightarrow}+\infty
$$

Theorem 1.2 follows, with

$$
t^{h}=h^{2+\alpha} \varepsilon\left(\log \frac{1}{\varepsilon}\right)^{\delta}=C h^{2 \sigma(d / 2-s)}\left(\log \frac{1}{h}\right)^{\delta} \underset{h \rightarrow 0}{\longrightarrow} 0 .
$$

\section{Proof of Corollary 1.4}

We argue by contradiction, by using a slight generalization of [7, Proposition 3.1].

Proposition 3.1. Let $d \geqslant 1, P: \mathbf{R}^{d} \rightarrow \mathbf{R}$. Suppose that there exist an admissible pair $(p, q)$, an index $k<2 / p=d / 2-d / q, T_{0}>0$, and a constant $C>0$ such that

$$
\left\|S(\cdot) u_{0}\right\|_{L^{p}\left(\left[0, T_{0}\right] ; L^{q}\left(\mathbf{R}^{d}\right)\right)} \leqslant C\left\|u_{0}\right\|_{H^{k}\left(\mathbf{R}^{d}\right)}, \quad \forall u_{0} \in H^{k}\left(\mathbf{R}^{d}\right) .
$$

Then for all

$$
k+\frac{d}{q}<s<\frac{d}{2}, \quad 0<\sigma<\frac{p}{2}
$$

and provided that either $\sigma$ is an integer, or there exists an integer $r$ such that $2 \sigma \geqslant r>d / 2$, the Cauchy problem for 1.1 is well posed in $H^{s}\left(\mathbf{R}^{d}\right)$. 
Since in Theorem 1.2 we can always consider $\sigma=1$, Theorem 1.2 and Proposition 3.1 imply Corollary 1.4 in the non-endpoint case $p>2$. The endpoint case then follows by interpolation with the case $(p, q)=(\infty, 2)$ : if an endpoint Strichartz estimate (with some loss) was available, then an non-endpoint would be as well.

ProOF. For $0<T \leqslant T_{0}$, introduce

$$
X_{T}=C\left([0, T] ; H^{s}\left(\mathbf{R}^{d}\right)\right) \cap L^{p}\left([0, T] ; W^{\ell, q}\left(\mathbf{R}^{d}\right)\right),
$$

where $\ell=s-k$. By assumption, $\ell>d / q$, so we have

$$
X_{T} \subset L^{p}\left([0, T] ; L^{\infty}\left(\mathbf{R}^{d}\right)\right) .
$$

This space is equipped with the norm

$$
\|u\|_{X_{T}}=\sup _{0 \leqslant t \leqslant T}\|u(t)\|_{H^{s}\left(\mathbf{R}^{d}\right)}+\left\|(1-\Delta)^{\ell / 2} u\right\|_{L^{p}\left([0, T] ; L^{q}\left(\mathbf{R}^{d}\right)\right)} .
$$

We construct the solution to 1.1 by a fixed point argument. Set

$$
\Phi(u)(t)=S(t) u_{0}-i \lambda \int_{0}^{t} S(t-\tau)\left(|u(\tau)|^{2 \sigma} u(\tau)\right) d \tau .
$$

We prove that for $T \in] 0, T_{0}$ ] sufficiently small, $\Phi$ is a contraction on some ball of $X_{T}$ centered at the origin. We have directly

$$
\|\Phi(u)\|_{C\left([0, T] ; H^{s}\left(\mathbf{R}^{d}\right)\right)} \lesssim\left\|u_{0}\right\|_{H^{s}\left(\mathbf{R}^{d}\right)}+\int_{0}^{t}\left\||u(\tau)|^{2 \sigma} u(\tau)\right\|_{H^{s}\left(\mathbf{R}^{d}\right)} d \tau .
$$

Now 3.1 implies the inhomogeneous estimate:

$$
\left\|\int_{0}^{t} S(t-\tau) f(\tau) d \tau\right\|_{L^{p}\left([0, T] ; L^{q}\left(\mathbf{R}^{d}\right)\right)} \lesssim\|f\|_{L^{1}\left([0, T] ; H^{k}\left(\mathbf{R}^{d}\right)\right)} .
$$

Indeed, setting $F_{\tau}(t)=\mathbf{1}_{\tau \leqslant t} S(t-\tau) f$, Minkowski inequality yields

$$
\left\|\int_{0}^{T} F_{\tau} d \tau\right\|_{L^{p}\left([0, T] ; L^{q}\left(\mathbf{R}^{d}\right)\right)} \leqslant \int_{0}^{T}\left\|F_{\tau}\right\|_{L^{p}\left([0, T] ; L^{q}\left(\mathbf{R}^{d}\right)\right)} d \tau \leqslant C \int_{0}^{T}\|f(\tau)\|_{H^{k}\left(\mathbf{R}^{d}\right)} d \tau .
$$

We infer

$$
\begin{aligned}
\|\Phi(u)\|_{X_{T}} & \lesssim\left\|u_{0}\right\|_{H^{s}\left(\mathbf{R}^{d}\right)}+\int_{0}^{t}\left\||u(\tau)|^{2 \sigma} u(\tau)\right\|_{H^{s}\left(\mathbf{R}^{d}\right)} d \tau \\
& \lesssim\left\|u_{0}\right\|_{H^{s}\left(\mathbf{R}^{d}\right)}+\int_{0}^{t}\|u(\tau)\|_{L^{\infty}\left(\mathbf{R}^{d}\right)}^{2 \sigma}\|u(\tau)\|_{H^{s}\left(\mathbf{R}^{d}\right)} d \tau \\
& \lesssim\left\|u_{0}\right\|_{H^{s}\left(\mathbf{R}^{d}\right)}+T^{\gamma}\|u\|_{L^{p}\left([0, T] ; L^{\infty}\left(\mathbf{R}^{d}\right)\right)}^{2 \sigma}\|u\|_{L^{\infty}\left([0, T] ; H^{s}\left(\mathbf{R}^{d}\right)\right)},
\end{aligned}
$$

with $\gamma=1-2 \sigma / p>0$. Therefore,

$$
\|\Phi(u)\|_{X_{T}} \leqslant C\|\| u_{0}\left\|_{H^{s}\left(\mathbf{R}^{d}\right)}+C T^{\gamma}\right\| u \|_{X_{T}}^{2 \sigma+1} .
$$

Similarly,

$$
\|\Phi(u)-\Phi(v)\|_{X_{T}} \leqslant C T^{\gamma}\left(\|u\|_{X_{T}}^{2 \sigma}+\|v\|_{X_{T}}^{2 \sigma}\right)\|u-v\|_{X_{T}} .
$$

This yields the local well-posedness result stated in Proposition 3.1 


\section{Ill-posedness}

The key remark is that all the cases of Proposition 1.5 boil down to an ordinary differential equation mechanism. Denote by $v$ the solution to

$$
i \partial_{t} v=\lambda|v|^{2 \sigma} v ; \quad v_{\mid t=0}=u_{0} .
$$

When $P(\xi)=c \cdot \xi$, we have

$$
u(t, x)=v(t, x+c t),
$$

and when $P(\xi)=c$, we have

$$
u(t, x)=v(t, x) e^{i c t},
$$

so it suffices to prove Proposition 1.5 in the case $P=0$. For fixed $x \in \mathbf{R}^{d}$,

$$
v(t, x)=u_{0}(x) e^{-i \lambda t\left|u_{0}(x)\right|^{2 \sigma}}
$$

is a solution; it is the only solution for which the multiplication $\bar{v} \times|v|^{2 \sigma} v$ is well-defined and real-valued, since then $i \bar{v} \partial_{t} v \in \mathbf{R}$, hence $\partial_{t}|v|^{2}=0$. The idea is then that $H^{d / 2}\left(\mathbf{R}^{d}\right)$ is not an algebra.

Consider

$$
u_{0}(x)=\delta \times\left(\log \frac{1}{|x|}\right)^{\alpha} \chi\left(|x|^{2}\right), \quad x \in \mathbf{R}^{d},
$$

with $\chi \in C_{0}^{\infty}(\mathbf{R}), \chi=1$ near the origin, and supp $\left.\chi \subset\right]-1,1[$. We compute

$$
\nabla v(t, x)=e^{-i \lambda t\left|u_{0}(x)\right|^{2 \sigma}} \nabla u_{0}(x)-2 i \sigma \lambda t\left|u_{0}(x)\right|^{2 \sigma} \nabla u_{0}(x) .
$$

We split the proof into three cases: for $d=2$, the proof is straightforward, for $d \geqslant 4$ even, the proof is similar but we omit the details of computations, and for $d$ odd, we simply sketch the argument.

Case $d=2$. First, $u_{0} \in H^{1}\left(\mathbf{R}^{2}\right)$ provided that $\alpha<1 / 2$. Now Proposition 1.5 follows if we can choose $\alpha<1 / 2$ so that $\left|u_{0}\right|^{2 \sigma} \nabla u_{0} \notin L^{2}\left(\mathbf{R}^{2}\right)$. Near the origin, we have, leaving out the constants,

$$
\left.\left.|| u_{0}(x)\right|^{2 \sigma} \nabla u_{0}(x)\right|^{2} \approx \frac{1}{|x|^{2}}(\log |x|)^{4 \alpha \sigma+2 \alpha-2} .
$$

The right hand side fails to be in $L_{\text {loc }}^{1}\left(\mathbf{R}^{2}\right)$ if we impose $4 \alpha \sigma+2 \alpha \geqslant 1$. So Proposition 1.5 follows, with

$$
\frac{1}{4 \sigma+2} \leqslant \alpha<\frac{1}{2}
$$

Case $d \geqslant 4$ even. The argument is the same as in the case $d=2$, with more computations that we simply sketch. We check by induction that for $k \geqslant 1$, there exist coefficients $\left(\beta_{j k}\right)_{1 \leqslant j \leqslant k}$ such that near the origin,

$$
\partial_{r}^{k} u_{0}(x)=\frac{1}{r^{k}} \sum_{j=1}^{k} \beta_{j k}\left(\log \frac{1}{|x|}\right)^{\alpha-j}, \quad \text { with } \beta_{1 k}=(-1)^{k-1}(k-1) ! \alpha .
$$

Therefore, the asymptotic behavior of $\partial_{r}^{k} u_{0}$ near the origin is given by:

$$
\partial_{r}^{k} u_{0}(x) \underset{r \rightarrow 0}{\sim}(-1)^{k-1}(k-1) ! \frac{\alpha}{r^{k}}\left(\log \frac{1}{|x|}\right)^{\alpha-1} .
$$


Like in the case $d=2, u_{0} \in H^{d / 2}\left(\mathbf{R}^{d}\right)$ provided that $\alpha<1 / 2$. We compute, for $t>0$, and as $x \rightarrow 0$ :

$$
\left|\partial_{r}^{k} v(t, x)\right|=\frac{1}{r^{k}}\left(c_{k}(t)\left(\log \frac{1}{|x|}\right)^{\gamma_{k}}+\mathcal{O}\left(\left(\log \frac{1}{|x|}\right)^{\gamma_{k}-\omega_{k}}\right)\right),
$$

with $c_{k}(t)>0, \omega_{k}>0$, and

$$
\gamma_{k}=\max ((2 \sigma k+1) \alpha-k,(2 \sigma+1) \alpha-1) .
$$

For $t>0, v(t, \cdot) \notin H^{d / 2}\left(\mathbf{R}^{d}\right)$ if, for $k=d / 2$, the first term in 4.3 is almost in $L_{\text {loc }}^{2}\left(\mathbf{R}^{d}\right)$, but not quite: we choose $\alpha$ so that $2 \gamma_{d / 2}=-1$. We find (like for $d=2$ )

$$
\alpha=\frac{1}{4 \sigma+2},
$$

which is consistent with the requirement $\alpha<1 / 2$. Thus, the first term in (4.3) is not in $L_{\text {loc }}^{2}\left(\mathbf{R}^{d}\right)$ due to a logarithmic divergence, while the remainder term is in $L_{\text {loc }}^{2}\left(\mathbf{R}^{d}\right)$, since $\omega_{k}>0$.

The case when $d$ is odd. We keep $u_{0}$ of the same form as in even dimensions, since we have found a value for $\alpha$ which does not depend on $d$ even:

$$
u_{0}(x)=\delta \times\left(\log \frac{1}{|x|}\right)^{1 /(4 \sigma+2)} \chi\left(|x|^{2}\right), \quad x \in \mathbf{R}^{d} .
$$

Recall the characterization of $H^{s}\left(\mathbf{R}^{d}\right)$ when $\left.s \in\right] 0,1\left[\right.$ : a function $f \in L^{2}\left(\mathbf{R}^{d}\right)$ belongs to $\left.H^{s}\left(\mathbf{R}^{d}\right), s \in\right] 0,1[$, if and only if

$$
\iint_{\mathbf{R}^{d} \times \mathbf{R}^{d}} \frac{|f(x)-f(x+y)|^{2}}{|y|^{d+2 s}} d x d y<\infty .
$$

When $d=1$, we check that $u_{0} \in H^{1 / 2}(\mathbf{R})$. We can also check that for $t>0, v(t, \cdot) \notin$ $H^{1 / 2}(\mathbf{R})$.

When $d \geqslant 3$, we compute $\partial_{r}^{k} u_{0}$ and $\partial_{r}^{k} v$ in the same fashion as above, and check that

$$
\nabla^{[d / 2]} u_{0} \in H^{1 / 2}\left(\mathbf{R}^{d}\right), \quad \text { and for } t>0, \quad \nabla^{[d / 2]} v(t, \cdot) \notin H^{1 / 2}\left(\mathbf{R}^{d}\right) .
$$

We leave out the details, since the technicalities are more involved than in the even dimensional case, and we believe that proving Proposition 1.5 in details is not worth such an effort.

Acknowledgement. The author is grateful to Valeria Banica for valuable comments on this work, and to the referee for pointing out some aspects which had to be clarified.

\section{References}

[1] R. ANTON, Strichartz inequalities for Lipschitz metrics on manifolds and nonlinear Schrödinger equation on domains, Bull. Soc. Math. France, 136 (2008), pp. 27-65.

[2] M. Ben-ARTZI, H. Koch, AND J.-C. SAUT, Dispersion estimates for fourth order Schrödinger equations, C. R. Acad. Sci. Paris Sér. I Math., 330 (2000), pp. 87-92.

[3] - Dispersion estimates for third order equations in two dimensions, Comm. Partial Differential Equations, 28 (2003), pp. 1943-1974.

[4] M. BEN-ARTZI AND J.-C. SAUT, Uniform decay estimates for a class of oscillatory integrals and applications, Differential Integral Equations, 12 (1999), pp. 137-145.

[5] M. Blair, H. Smith, And C. Sogge, On Strichartz estimates for Schrödinger operators in compact manifolds with boundary, Proc. Amer. Math. Soc., 136 (2008), pp. 247-256.

[6] J. Bona AND J.-C. SAUT, Dispersive blow-up II. Schrödinger-type equations, optical and oceanic rogue waves, Chin. Ann. Math. Ser. B, 31 (2010), pp. 793-818. 
[7] N. BURQ, P. GÉRARD, AND N. TZVETKOv, Strichartz inequalities and the nonlinear Schrödinger equation on compact manifolds, Amer. J. Math., 126 (2004), pp. 569-605.

[8] - Multilinear eigenfunction estimates and global existence for the three dimensional nonlinear Schrödinger equations, Ann. Sci. École Norm. Sup. (4), 38 (2005), pp. 255-301.

[9] T. CAZEnave, D. FAnG, AND Z. HAN, Continuous dependence for NLS in fractional order spaces, Ann. Inst. H. Poincaré Anal. Non Linéaire, 28 (2011), pp. 135-147.

[10] T. CAZENAVE AND F. WeISSLER, The Cauchy problem for the critical nonlinear Schrödinger equation in $H^{s}$, Nonlinear Anal., 14 (1990), pp. 807-836.

[11] M. Christ, J. COlliander, AND T. TAO, Ill-posedness for nonlinear Schrödinger and wave equations. Archived as http://arxiv.org/abs/math/0311048

[12] M. Colin AND D. LANNES, Short pulses approximations in dispersive media, SIAM J. Math. Anal., 41 (2009), pp. 708-732.

[13] S. CUI, Pointwise estimates for a class of oscillatory integrals and related $L^{p}-L^{q}$ estimates, J. Fourier Anal. Appl., 11 (2005), pp. 441-457.

[14] - Pointwise estimates for oscillatory integrals and related $L^{p}-L^{q}$ estimates. II. Multidimensional case, J. Fourier Anal. Appl., 12 (2006), pp. 605-627.

[15] S. CUI AND C. GuO, Well-posedness of higher-order nonlinear Schrödinger equations in Sobolev spaces $H^{s}\left(\mathbb{R}^{n}\right)$ and applications, Nonlinear Anal., 67 (2007), pp. 687-707.

[16] A. Debussche And E. Faou, Modified energy for split-step methods applied to the linear Schrödinger equation, SIAM J. Numer. Anal., 47 (2009), pp. 3705-3719.

[17] J. Ginibre And G. Velo, Generalized Strichartz inequalities for the wave equation, J. Funct. Anal., 133 (1995), pp. 50-68.

[18] N. HAYASHI AND P. NAUMKIN, Asymptotic properties of solutions to dispersive equation of Schrödinger type, J. Math. Soc. Japan, 60 (2008), pp. 631-652.

[19] T. Kato, On nonlinear Schrödinger equations, Ann. IHP (Phys. Théor.), 46 (1987), pp. 113-129.

[20] C. Kenig, G. PONCE, AND L. VEGA, Oscillatory integrals and regularity of dispersive equations, Indiana Univ. Math. J., 40 (1991), pp. 33-69.

[21] — Higher-order nonlinear dispersive equations, Proc. Amer. Math. Soc., 122 (1994), pp. 157-166.

[22] — On the ill-posedness of some canonical dispersive equations, Duke Math. J., 106 (2001), pp. $617-$ 633.

[23] D. LANNES, High frequency nonlinear optics: from the nonlinear Schrödinger approximation to ultrashort pulses equations, Proc. Roy. Soc. Edinburgh Sect. A, 141 (2011), pp. 253-286.

[24] M. NAKAMURA AND T. OZAWA, Nonlinear Schrödinger equations in the Sobolev space of critical order, J. Funct. Anal., 155 (1998), pp. 364-380.

[25] B. PAUSADER, Global well-posedness for energy critical fourth-order Schrödinger equations in the radial case, Dyn. Partial Differ. Equ., 4 (2007), pp. 197-225.

[26] - The cubic fourth-order Schrödinger equation, J. Funct. Anal., 256 (2009), pp. 2473-2517.

[27] T. TAO, Nonlinear dispersive equations, vol. 106 of CBMS Regional Conference Series in Mathematics, Published for the Conference Board of the Mathematical Sciences, Washington, DC, 2006. Local and global analysis.

[28] Y. TSUTSUMI, $L^{2}$-solutions for nonlinear Schrödinger equations and nonlinear groups, Funkcial. Ekvac., 30 (1987), pp. 115-125.

Univ. Montpellier 2, Mathématiques, CC 051, F-34095 Montpellier; And CNRS, UMR 5149, F-34095 MONTPELLIER, FRANCE

E-mail address: Remi.Carles@math.cnrs.fr 